\title{
The Chemical Evolution of Magnesium Isotopic Abundances in the Solar Neighbourhood
}

\author{
Y. Fenner ${ }^{1,2}$, B. K. Gibson ${ }^{1}$, H.-c. Lee ${ }^{1}$, A. I. Karakas ${ }^{3}$, J. C. Lattanzio ${ }^{3}$, \\ A. Chieffi ${ }^{1,3,4}$, M. Limongi ${ }^{1,3,5}$ and D. Yong 6 \\ ${ }^{1}$ Centre for Astrophysics and Supercomputing, Swinburne University, Hawthorn, Victoria 3122, Australia \\ yfenner@astro.swin.edu.au \\ ${ }^{2}$ UCO/Lick Observatory, University of California Santa Cruz, Santa Cruz, California 95064, USA \\ ${ }^{3}$ Centre for Stellar and Planetary Astrophysics, Monash University, Victoria 3800, Australia \\ ${ }^{4}$ Istituto di Astrofisica Spaziale e Fisica Cosmica, Via Fosso del Cavaliere, I-00133, Roma, Italy \\ ${ }^{5}$ Istituto Nazionale di Astrofisica — Osservatorio Astronomico di Roma, Via Frascati 33, I-00040, \\ Monteporzio Catone, Italy \\ ${ }^{6}$ Department of Astronomy, University of Texas, Austin TX 78712, USA
}

Received 2003 June 10, accepted 2003 July 23

\begin{abstract}
The abundance of the neutron-rich magnesium isotopes observed in metal-poor stars is explained quantitatively with a chemical evolution model of the local Galaxy that considers - for the first time the metallicity-dependent contribution from intermediate mass stars. Previous models that simulate the variation of $\mathrm{Mg}$ isotopic ratios with metallicity in the solar neighbourhood have attributed the production of ${ }^{25} \mathrm{Mg}$ and ${ }^{26} \mathrm{Mg}$ exclusively to hydrostatic burning in massive stars. These models match the data well for $[\mathrm{Fe} / \mathrm{H}]>-1.0$ but severely underestimate ${ }^{25,26} \mathrm{Mg} /{ }^{24} \mathrm{Mg}$ at lower metallicities. Earlier studies have noted that this discrepancy may indicate a significant role played by intermediate mass stars. Only recently have detailed calculations of intermediate mass stellar yields of ${ }^{25} \mathrm{Mg}$ and ${ }^{26} \mathrm{Mg}$ become available with which to test this hypothesis. In an extension of previous work, we present a model that successfully matches the $\mathrm{Mg}$ isotopic abundances in nearby Galactic disk stars through the incorporation of nucleosynthesis predictions of $\mathrm{Mg}$ isotopic production in asymptotic giant branch stars.
\end{abstract}

Keywords: Galaxy: evolution — stars: abundances

\section{Introduction}

Magnesium is one of the few elements for which the stellar abundance of individual isotopes can be reliably measured. The relative abundances of $\mathrm{Mg}$ isotopes provide a useful probe into the nucleosynthesis history of the Milky Way because they have their origin in different classes of stars. According to standard theories of stellar evolution, most of the $\mathrm{Mg}$ isotopes originate from massive stars. The heavy $\mathrm{Mg}$ isotopes behave as secondary elements inside massive stars and their production scales with the number of initial 'seed' nuclei. Consequently, very little ${ }^{25,26} \mathrm{Mg}$ is synthesised by massive stars until an initial $[\mathrm{Fe} / \mathrm{H}]$ of $\sim-1$ is reached, whereas the generation of ${ }^{24} \mathrm{Mg}$ operates fairly independently of initial metallicity.

${ }^{25} \mathrm{Mg}$ and ${ }^{26} \mathrm{Mg}$ are detected in metal-poor stars in higher proportions than one would expect if these neutronrich isotopes originated exclusively from massive stars (Gay \& Lambert 2000). Detailed models of the evolution of $\mathrm{Mg}$ isotopic ratios find that massive stars alone are insufficient to account for the values of ${ }^{25,26} \mathrm{Mg} /{ }^{24} \mathrm{Mg}$ at low $[\mathrm{Fe} / \mathrm{H}]$, hinting at an additional production site (Timmes, Woosley, \& Weaver 1995; Goswami \& Prantzos 2000; Alibes, Labay, \& Canal 2001).

Karakas \& Lattanzio (2003a, 2003b) have shown that ${ }^{25} \mathrm{Mg}$ and ${ }^{26} \mathrm{Mg}$ production is substantial in metal-poor intermediate mass stars (IMS). In the low-metallicity regime, asymptotic giant branch (AGB) stars are believed to generate ${ }^{25} \mathrm{Mg}$ and ${ }^{26} \mathrm{Mg}$ from alpha capture onto ${ }^{22} \mathrm{Ne}$ triggered by He shell thermal pulsing. More massive AGB stars $\left(4<m / \mathbf{M}_{\odot}<6\right)$ are less common than lower mass stars but may be a significant production site for ${ }^{25} \mathrm{Mg}$ and ${ }^{26} \mathrm{Mg}$. Temperatures at the base of the convective envelope in these stars can be high enough to burn ${ }^{24} \mathrm{Mg}$ via hot bottom burning as well as synthesise large amounts of ${ }^{25} \mathrm{Mg}$ and ${ }^{26} \mathrm{Mg}$. We explore the possibility that AGB stars produce sufficient quantities of ${ }^{25} \mathrm{Mg}$ and ${ }^{26} \mathrm{Mg}$ to resolve the discrepancy between observations and previous model predictions.

\section{The Chemical Evolution Model}

The temporal and radial evolution of the isotopic abundances in the Milky Way was calculated under the assumption that the Galaxy formed via the accretion of gas at a rate decreasing exponentially with time. For the sake of simplicity, we assumed only a single episode of primordial gas accretion, with a timescale of $7 \mathrm{Gyr}$ at the solar radius. However the results were not significantly different for a two-phase accretion model. We traced the chemical elements through the ongoing cycles of star formation, nucleosynthesis, and ejection into the interstellar medium (ISM) via supernovae ( $\mathrm{SNe}$ ) explosions and stellar winds. In order to precisely monitor the abundances of isotopes with different production sites, mass- and metallicitydependent stellar lifetimes and yields were employed. 
The rate of star formation in this model varies with the square of the gas surface density and inversely with Galactocentric radius. This type of radially-dependent law is motivated by the idea that spiral arm patterns trigger star formation (e.g. Prantzos \& Silk 1998). The mass distribution of each new generation of stars was governed by the Kroupa, Tout, \& Gilmore (1993) three-component initial mass function (IMF), with lower and upper mass limits of 0.8 and $100 \mathrm{M}_{\odot}$, respectively.

Three basic models were constructed, differing only in the adopted nucleosynthesis prescriptions. Firstly, $L S C+A G B$ refers to a combination of low and intermediate mass stellar yields from Karakas \& Lattanzio (2003a, 2003b) and a grid of mass and metallicity dependent $\left(Z=0,10^{-3}\right.$, and 0.02$)$ massive star yields provided by M. Limongi (2001, private communication), calculated using the FRANEC code described in Limongi, Straneiro, \& Chieffi (2000) and Limongi \& Chieffi (2002). The second model, $L S C$ no $A G B$, is identical to the first model but ignores the AGB contribution to ${ }^{25} \mathrm{Mg}$ and ${ }^{26} \mathrm{Mg}$ production. Finally, the model $W W$ no $A G B$ replicates $L S C$ no $A G B$ but using metallicity-dependent Woosley $\&$ Weaver (1995) yields for massive stars. All models adopt yields for Type Ia SNe from the W7 model of Iwamoto et al. (1999). The SNe Ia contribution to chemical evolution was calculated following the method from Matteucci \& Greggio (1986). It was assumed that $3 \%$ of binaries culminate in $\mathrm{SNe}$ Ia, since this fraction provides a good fit to the present-day SNe Ia rate (e.g. Alibes et al. 2001). For stars whose metallicity lies below (above) the range covered by the nucleosynthesis models we estimate their yields by extrapolating from the two lowest (highest) metallicity grids.

The Karakas \& Lattanzio (2003a, 2003b) stellar models comprise a grid of $\mathrm{Mg}$ isotopic yields covering a range of low to intermediate stellar mass $\left(1 \leq m / \mathrm{M}_{\odot} \leq 6\right)$ and a variety of compositions $(Z=0.004,0.008$, and 0.02 , supplemented by an unpublished 0.0001 grid calculated with the same code) that is well suited for chemical evolution models. These models have been evolved from the premain sequence to near the end of the thermal-pulsing AGB phase. The nucleosynthesis calculations are performed separately to determine the production of the isotopes.

\section{Results and Discussion}

Figure 1 shows the predicted evolution of magnesium isotopic ratios with $[\mathrm{Fe} / \mathrm{H}]$ from the models $L S C+A G B$ (solid line) and $L S C$ no $A G B$ (dotted line) for the solar region. ${ }^{25} \mathrm{Mg} /{ }^{24} \mathrm{Mg}$ and ${ }^{26} \mathrm{Mg} /{ }^{24} \mathrm{Mg}$ are shown in the upper and lower panels, respectively, along with measured abundance ratios in local dwarfs from Gay \& Lambert (2000) and cool subdwarfs from D. Yong (in preparation). Representative observational errors are indicated by the large crosses. Although the quoted errors for ${ }^{25} \mathrm{Mg}$ and ${ }^{26} \mathrm{Mg}$ are identical in both studies, ${ }^{26} \mathrm{Mg}$ is expected to be more accurately determined than ${ }^{25} \mathrm{Mg}$ because the ${ }^{26} \mathrm{MgH}$ line is less blended with ${ }^{24} \mathrm{MgH}$. Both models shown in Figure 1 predict ratios larger than solar (indicated by squares) but
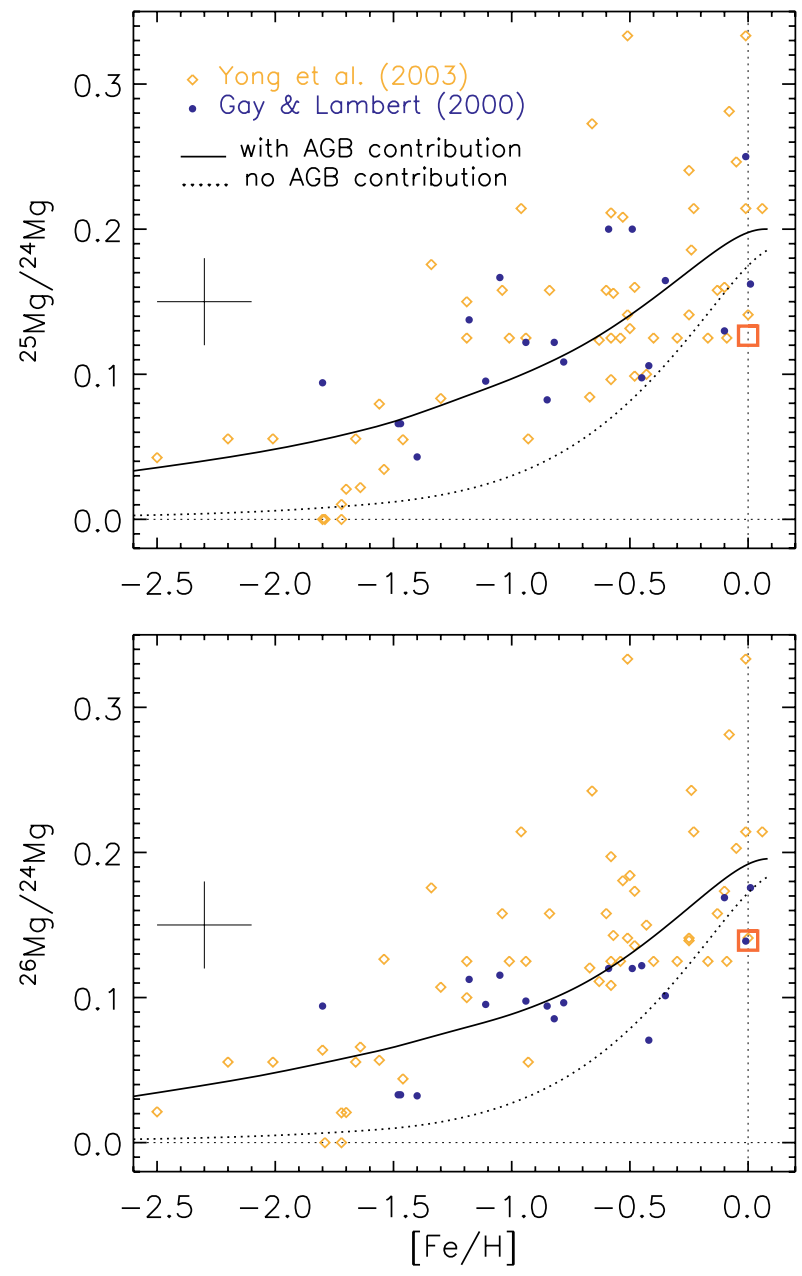

Figure 1 Variation of magnesium isotopic ratios with metallicity $[\mathrm{Fe} / \mathrm{H}] .{ }^{25} \mathrm{Mg} /{ }^{24} \mathrm{Mg}$ and ${ }^{26} \mathrm{Mg} /{ }^{24} \mathrm{Mg}$ are shown in the upper and lower panels, respectively. Circles correspond to stellar abundances observed by Gay \& Lambert (2000), while diamonds represent a sample of halo and thick disk stars from D. Yong (in preparation). The large cross appearing in both panels indicates typical observational errors. Solar values appear as squares. The predicted trend of our solar neighbourhood model incorporating $\mathrm{Mg}$ isotopic yields from AGBs (solid line) is shown against a model without the AGB contribution (dotted line). Both models arrive at similar present-day values, however only the AGB model matches the empirical data at low metallicities.

consistent with the data of Gay \& Lambert (2000). It is not surprising that the models reach similar present-day values irrespective of whether AGB stars are included, because massive stars are responsible for most of the neutron-rich $\mathrm{Mg}$ isotopes in the present-day ISM. In this model $10 \%$ of ${ }^{26} \mathrm{Mg}$ present in the ISM at $[\mathrm{Fe} / \mathrm{H}]=0$ comes from AGB stars compared with $\sim 70 \%$ at $[\mathrm{Fe} / \mathrm{H}]=-1$ and nearly $90 \%$ at $[\mathrm{Fe} / \mathrm{H}]=-2$.

The dotted lines reveal that below $[\mathrm{Fe} / \mathrm{H}] \sim-1$, massive stars alone seriously underproduce ${ }^{25} \mathrm{Mg}$ and ${ }^{26} \mathrm{Mg}$ with respect to ${ }^{24} \mathrm{Mg}$. Much better agreement is obtained by including the contribution from AGB stars. In particular, most of the heavy $\mathrm{Mg}$ isotopic abundance at low metallicity is controlled by the $4-6 \mathrm{M}_{\odot}$ stars that undergo hot bottom burning and whose He shells are hot enough to trigger the ${ }^{22} \mathrm{Ne}(\alpha, n)^{25} \mathrm{Mg}$ and ${ }^{22} \mathrm{Ne}(\alpha, \gamma)^{26} \mathrm{Mg}$ reactions 
(Karakas \& Lattanzio 2003a, 2003b). These stars typically have lifetimes between 60 and $170 \mathrm{Myr}$.

The high abundances of ${ }^{25} \mathrm{Mg}$ and ${ }^{26} \mathrm{Mg}$ from the D. Yong (in preparation) dataset pose more of a challenge and are difficult to reconcile with our models, even with an AGB contribution. These stars were selected by their large transverse velocities as part of an effort to compile a sample of metal-poor unevolved subdwarfs (Yong \& Lambert 2003). Based on their high reduced proper motion, it is likely that most of the sample is distinct from the thin disk, belonging instead to the halo at low metallicities or the thick disk at higher metallicities. One might expect that stars enhanced in ${ }^{25} \mathrm{Mg}$ and ${ }^{26} \mathrm{Mg}$ are also either (1) rich in s-process elements if they have been heavily contaminated by AGB stars, or (2) $\alpha$-enhanced if they belong to the halo/thick disk. D. Yong (in preparation) reports that there does not appear to be an obvious relationship between high values of ${ }^{25,26} \mathrm{Mg}$ and the abundance of s-process or $\alpha$-elements.

The predicted trend of ${ }^{26} \mathrm{Mg} /{ }^{24} \mathrm{Mg}$ with $[\mathrm{Fe} / \mathrm{H}]$ is plotted in Figure 2 at different Galactic radii for the $L S C+A G B$ model. The dotted curve corresponds to the innermost radius of the model $(4 \mathrm{kpc})$ and the dashed curve to the outermost radius $(16 \mathrm{kpc})$. The exponential timescale for infalling gas was assumed to increase linearly with radius, taking on a value of $7 \mathrm{Gyr}$ at the solar radius. Due to this 'inside-out' accretion scenario and the radially-dependent star formation law, the timescale of star formation is much longer in the outer disk. Only ${ }^{26} \mathrm{Mg} /{ }^{24} \mathrm{Mg}$ is plotted because the ${ }^{25} \mathrm{MgH}$ line is less reliably determined than the ${ }^{26} \mathrm{MgH}$ line due to more severe blending with the ${ }^{24} \mathrm{MgH}$ feature (Gay \& Lambert 2000). This model predicts a greater spread in ${ }^{26} \mathrm{Mg} /{ }^{24} \mathrm{Mg}$ in metal-poor stars versus metal-rich stars throughout the Galaxy. The dispersion in the metal-poor regime reflects the dependence of ${ }^{25} \mathrm{Mg}$ and ${ }^{26} \mathrm{Mg}$ abundance on the timescale of star formation. Since the lowest metallicity AGB models predict the highest ${ }^{25} \mathrm{Mg}$ and ${ }^{26} \mathrm{Mg}$ yields, environments such as the outer disk, with protracted star formation and slowly increasing metallicity over time, encourage generations of IMS to elevate ${ }^{25} \mathrm{Mg} /{ }^{24} \mathrm{Mg}$ and ${ }^{26} \mathrm{Mg} /{ }^{24} \mathrm{Mg}$. The inner Galaxy, in contrast, is believed to have experienced intense star formation very early in its history, rapidly enriching the ISM with metals. Once $[\mathrm{Fe} / \mathrm{H}]$ exceeds about -1 , massive stars begin to surpass IMS as the chief source of neutron-rich $\mathrm{Mg}$ isotopes. The spread becomes smaller for $[\mathrm{Fe} / \mathrm{H}]>-1$, at which point metallicity rather than star formation history drives ${ }^{26} \mathrm{Mg} /{ }^{24} \mathrm{Mg}$.

Figure 3 illustrates the lack of dispersion across different radii for the $L S C$ no $A G B$ model. There is some scatter in ${ }^{26} \mathrm{Mg} /{ }^{24} \mathrm{Mg}$ at high $[\mathrm{Fe} / \mathrm{H}]$ that stems from the delayed release of iron from Type Ia SNe. As most of the ejecta from $\mathrm{SNe} I \mathrm{I}$ is in the form of iron, these events increase $[\mathrm{Fe} / \mathrm{H}]$ to a greater extent than overall metallicity. A consequence of the characteristic SNe Ia time delay of about $1 \mathrm{Gyr}$ is that for the same abundance of iron, the inner disk is expected to be $\alpha$-enhanced and therefore

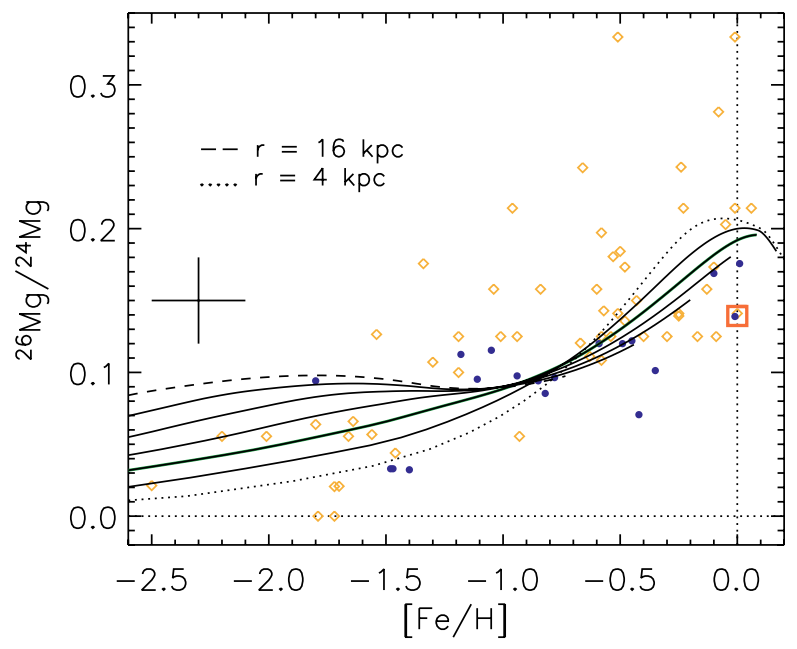

Figure 2 Evolution of ${ }^{26} \mathrm{Mg} /{ }^{24} \mathrm{Mg}$ as a function of $[\mathrm{Fe} / \mathrm{H}]$ at different Galactic radii. Symbols have the same meaning as in Figure 1. The curves correspond to predicted behaviour at seven evenly spaced radii, ranging from 4 to $16 \mathrm{kpc}$ for the $L S C+A G B$ model. The green line indicates the solar radius $(8 \mathrm{kpc})$.

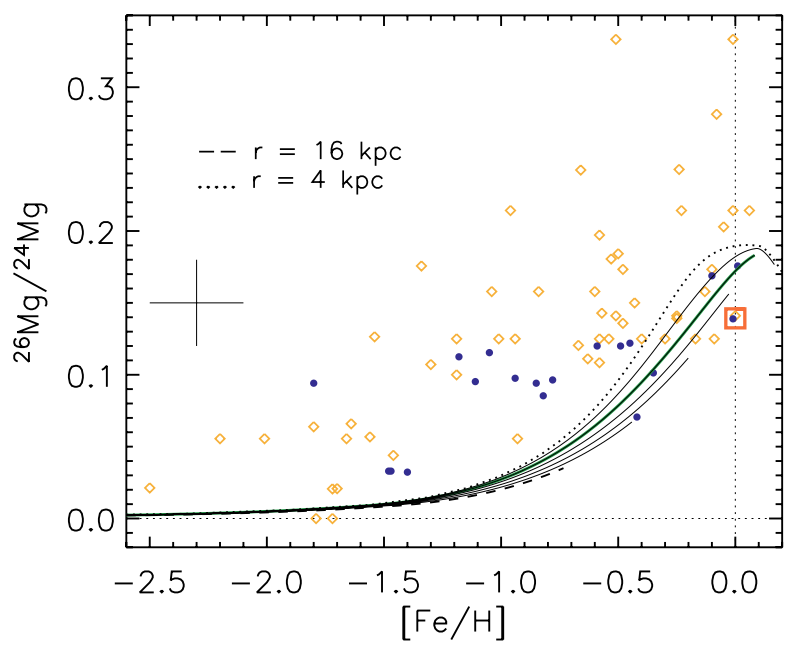

Figure 3 Same as Figure 2, but for model LSC no AGB (see text for details).

have greater total abundance of nuclei than the outer disk. With more seed nuclei, the inner disk for a given value of $[\mathrm{Fe} / \mathrm{H}]$ should see higher values of ${ }^{26} \mathrm{Mg} /{ }^{24} \mathrm{Mg}$ than the outer disk. Precisely this effect is revealed in Figure 3.

For the sake of comparing how robust our results are to explosive $\mathrm{SNe}$ yields from different authors, we plot in Figure 4 the predicted evolution of ${ }^{25} \mathrm{Mg} /{ }^{24} \mathrm{Mg}$ and ${ }^{26} \mathrm{Mg} /{ }^{24} \mathrm{Mg}$ for the solar neighbourhood for the $L S C$ no $A G B$ and $W W$ no $A G B$ models. There is excellent agreement between the two models, although Woosley \& Weaver's (1995) nucleosynthesis models predict about $20 \%$ less ${ }^{25} \mathrm{Mg}$ than ${ }^{26} \mathrm{Mg}$, while Limongi et al. (2000) and Limongi \& Chieffi (2002) produce these isotopes in roughly equal numbers. The $\mathrm{Mg}$ isotopic yields depend on the adopted ${ }^{12} \mathrm{C}(\alpha, \gamma){ }^{16} \mathrm{O}$ rate (Imbriani et al. 2001). Specifically, lowering the value of ${ }^{12} \mathrm{C}(\alpha, \gamma){ }^{16} \mathrm{O}$ leads to higher final yields of ${ }^{24} \mathrm{Mg}$ but leaves the heavier $\mathrm{Mg}$ isotopes relatively uneffected. The Limongi et al. (2000) 


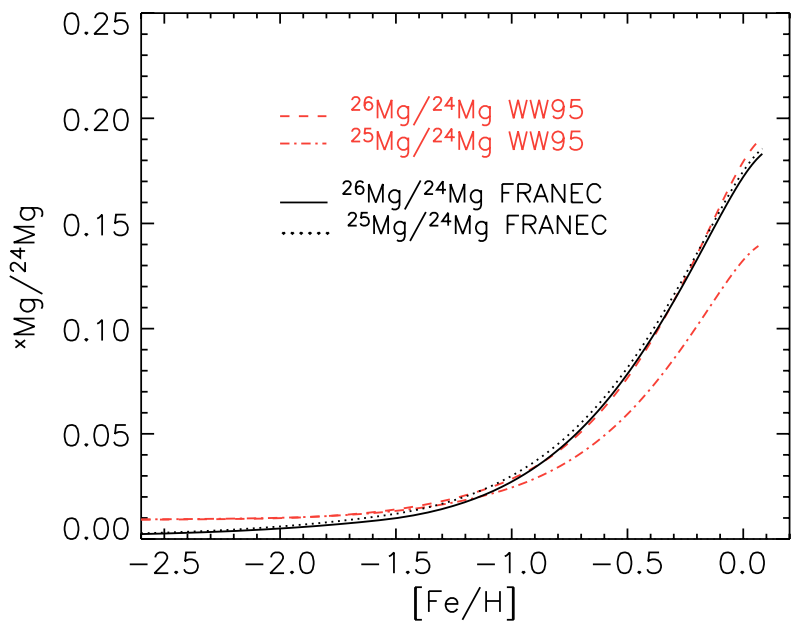

Figure 4 Evolution of magnesium isotopic ratios for models using different nucleosynthetic prescriptions for massive star yields. Red lines denote massive star yields from Woosley \& Weaver (1995) and black lines denote yields from Limongi et al. (2000) and Limongi \& Chieffi (2002). The AGB contribution has been ignored for the sake of comparison. There is good agreement between the two sets of yields.

and Limongi \& Chieffi (2002) yields employed in this Milky Way model use a large ${ }^{12} \mathrm{C}(\alpha, \gamma){ }^{16} \mathrm{O}$ rate, hence produce low ${ }^{24} \mathrm{Mg}$ yields. The ${ }^{25} \mathrm{Mg} /{ }^{24} \mathrm{Mg}$ and ${ }^{26} \mathrm{Mg} /{ }^{24} \mathrm{Mg}$ ratios could be decreased with a lower ${ }^{12} \mathrm{C}(\alpha, \gamma){ }^{16} \mathrm{O}$ value such as that from Kunz et al. (2002) which is used in the latest version of the FRANEC code (Limongi \& Chieffi 2003). In this case, the $\mathrm{Mg}$ isotopic ratios could be reconciled with solar values, but at the expense of obtaining a good fit to the Gay \& Lambert (2000) data.

One of the greatest uncertainties in the model concerns the precise form of the stellar IMF. While most elemental yields are fairly robust to changes in the slope and limits of the IMF, this is not the case for the heavy Mg isotopes, whose production in solar metallicity massive stars rises very sharply with stellar mass. Thus, the most massive stars play a crucial role in determining ${ }^{25} \mathrm{Mg} /{ }^{24} \mathrm{Mg}$ and ${ }^{26} \mathrm{Mg} /{ }^{24} \mathrm{Mg}$ despite being vastly outnumbered by lower mass stars. Figure 5 displays the yield of ${ }^{26} \mathrm{Mg}$ relative to ${ }^{24} \mathrm{Mg}$ as a function of initial mass for the solar metallicity models of Karakas \& Lattanzio (2003a, 2003b), Limongi et al. (2000), and Limongi \& Chieffi (2002) (solid line). The solar metallicity massive star yields extend only as far as $35 \mathrm{M}_{\odot}$ and were linearly extrapolated up to $100 \mathrm{M}_{\odot}$. The largest mass in the AGB models is $6 \mathrm{M}_{\odot}$. For most elements, including $\mathrm{Fe}$ and ${ }^{24} \mathrm{Mg}$, the yields between $6-8 \mathrm{M}_{\odot}$ were derived by extrapolating up from the AGB models. However, we conservatively assumed that the $6-8 \mathrm{M}_{\odot}$ stars eject the same quantity of ${ }^{25} \mathrm{Mg}$ and ${ }^{26} \mathrm{Mg}$ as the $6 \mathrm{M}_{\odot}$ star. This causes a dip in ${ }^{26} \mathrm{Mg} /{ }^{24} \mathrm{Mg}$ at $8 \mathrm{M}_{\odot}$. Superimposed on the same figure are IMFs from Salpeter (1955) (dotted line) and Kroupa et al. (1993) (dashed line) normalised over the mass range $0.08-100 \mathrm{M}_{\odot}$. The Salpeter IMF has the form of a single slope power law (this model uses an index of 1.31) and places a higher proportion of the mass of a stellar generation into both the lower and upper

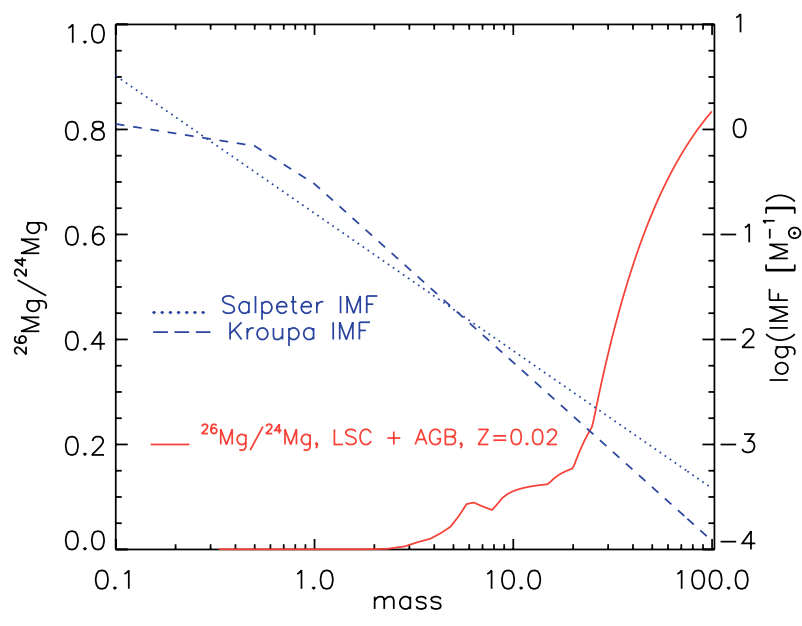

Figure 5 Yield of ${ }^{26} \mathrm{Mg} /{ }^{24} \mathrm{Mg}$ vs initial stellar mass for solar metallicity nucleosynthesis models (solid line). Yields for low- and intermediate mass stars are from Karakas \& Lattanzio (2003a, 2003b), and from Limongi et al. (2000) and Limongi \& Chieffi (2002) for massive stars. The dotted line corresponds to the Salpeter (1955) IMF, and the dashed line indicates the Kroupa et al. (1993) IMF. Both functions are normalised to unity over the mass range $0.08-100 \mathrm{M}_{\odot}$ (the scale appears on the right-hand vertical axis).

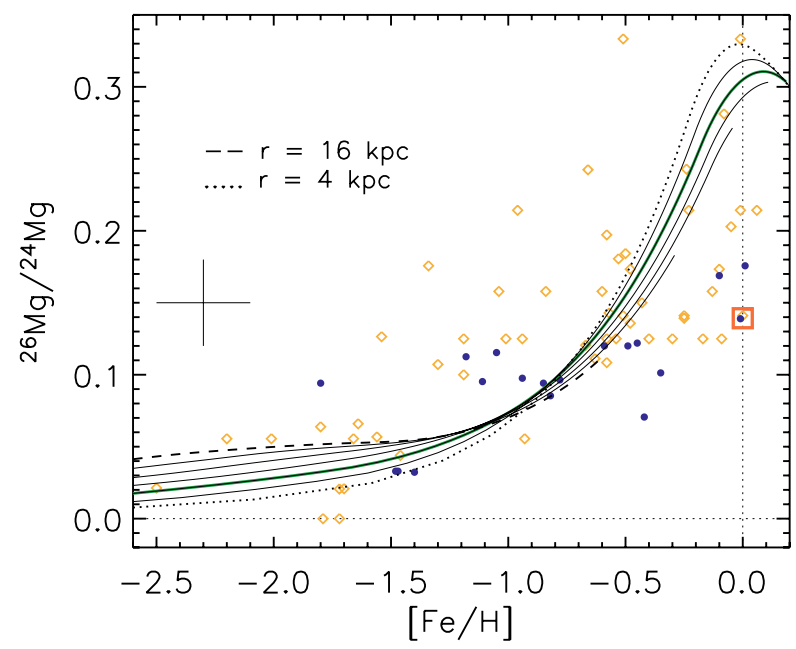

Figure 6 Same as Figure 2 but for the case of a single component Salpeter (1955) IMF in place of Kroupa et al. (1993).

extremes of the mass distribution when compared with the Kroupa et al. (1993) IMF. It is apparent that the role of stars between $0.3 \geq m / \mathrm{M}_{\odot} \geq 6$ is emphasised by adopting a Kroupa et al. (1993) IMF, whereas the frequency of high mass stars is increased with the Salpeter IMF.

One can anticipate that a Kroupa et al. (1993) IMF would give rise to more AGB stars than a Salpeter (1955) function, leading to higher values of ${ }^{26} \mathrm{Mg} /{ }^{24} \mathrm{Mg}$ at low metallicity. At metallicities approaching solar, however, the Salpeter IMF should generate the highest ${ }^{26} \mathrm{Mg} /{ }^{24} \mathrm{Mg}$ ratios, since it favours the birth of massive stars when compared with the Kroupa et al. (1993) law. The influence of the IMF can be seen by comparing the results presented in Figure 6, which were derived using the Salpeter function, with those from Figure 2. A ratio of ${ }^{26} \mathrm{Mg} /{ }^{24} \mathrm{Mg} \sim 0.3$ at $[\mathrm{Fe} / \mathrm{H}]=0$ is obtained with a Salpeter IMF. This is $\sim 50 \%$ 
higher than in the Kroupa et al. (1993) case and over twice the solar value. Owing to the production of fewer AGB stars, ${ }^{26} \mathrm{Mg} /{ }^{26} \mathrm{Mg}$ at $[\mathrm{Fe} / \mathrm{H}]=-2$ in the Salpeter case is about half the value derived with the Kroupa et al. (1993) IMF. The Kroupa et al. (1993) model is in better agreement with the Gay \& Lambert (2000) dataset. Although the large values of ${ }^{26} \mathrm{Mg} /{ }^{24} \mathrm{Mg}$ measured by D. Yong (in preparation) in higher metallicity stars could be attained by increasing the role of massive stars, this comes at the expense of satisfying observations at low $[\mathrm{Fe} / \mathrm{H}]$. Multicomponent IMFs with steeper slopes at high mass are favoured over the Salpeter single power law on both observational and theoretical grounds (e.g. Scalo 1986; Kroupa et al. 1993).

It has been suggested that the mass distribution of a stellar generation is influenced by factors including the thermal energy and chemical composition of the starforming gas (Larson 1998). Accordingly, the IMF might be expected to evolve over time. There are theoretical arguments that the IMF of primordial gas would be biased toward higher mass stars, while higher metallicity environments would form relatively more low mass stars (Kroupa 2001). If the IMF followed this trend then the fit between our model predictions and empirical constraints would worsen. At present, however, the observational evidence for a variable IMF is not overwhelming.

\section{Conclusions}

Thermally pulsing AGB stars are shown to be excellent candidates for the additional production site of neutronrich $\mathrm{Mg}$ isotopes needed to account for observations at low metallicities. The failure of previous chemical evolution models to match ${ }^{25} \mathrm{Mg} /{ }^{24} \mathrm{Mg}$ and ${ }^{26} \mathrm{Mg} /{ }^{24} \mathrm{Mg}$ observed in local metal-poor stars may be resolved if intermediate mass stars produce ${ }^{25} \mathrm{Mg}$ and ${ }^{26} \mathrm{Mg}$ in quantities given by the calculations of Karakas \& Lattanzio (2003a, 2003b).

According to the chemical evolution model presented in this paper, massive stars are responsible for most of the heavy $\mathrm{Mg}$ isotopes in the present-day ISM, but played a secondary role to $4-6 \mathrm{M}_{\odot} \mathrm{AGB}$ stars at earlier epochs.
A consequence of this model is that the spread in ${ }^{25} \mathrm{Mg} /{ }^{24} \mathrm{Mg}$ and ${ }^{26} \mathrm{Mg} /{ }^{24} \mathrm{Mg}$ should be greater at low metallicities across the Milky Way, although this conclusion was shown to be sensitive to the adopted IMF. While the high ratios of ${ }^{25} \mathrm{Mg} /{ }^{24} \mathrm{Mg}$ and ${ }^{26} \mathrm{Mg} /{ }^{24} \mathrm{Mg}$ in D. Yong's (in preparation) halo and thick disk sample remain a mystery and warrant further investigation, our model provides an excellent match to the measurements of local stars from Gay \& Lambert (2000).

\section{Acknowledgments}

The financial support of the Australian Research Council (through its Large Grant, Discovery Project, and Linkage International schemes) and the Victorian Partnership for Advanced Computing (through its Expertise Grants scheme) is acknowledged.

\section{References}

Alibes, A., Labay, J., \& Canal, R. 2001, A\&A, 370, 1103

Gay, P. L., \& Lambert, D. L. 2000, ApJ, 533, 260

Goswami, A., \& Prantzos, N. 2000, A\&A, 359, 191

Imbriani, G., Limongi, M., Gialanella, L., Terrasi, F., Straniero, O., \& Chieffi, A. 2001, ApJ, 558, 903

Iwamoto, K., Brachwitz, F., Nomoto, K., Kishimoto, N., Umeda, H., Hix, W. R., \& Thielemann, F.-K. 1999, ApJS, 125, 439

Karakas, A. I., \& Lattanzio, J. C. 2003a, Origin and Evolution of the Elements, Carnegie Observatories Astrophysics Series Volume 4, ed. A. McWilliam \& M. Rauch (Pasadena: Carnegie Observatories), http://www.ociw.edu/ociw/symposia/series/symposium-4/ proceedings.html

Karakas, A. I., \& Lattanzio, J. C. 2003b, PASA, 20, 279

Kroupa, P., Tout, C. A., \& Gilmore, G. 1993, MNRAS, 262, 545

Kroupa, P. 2001, MNRAS, 322, 231

Kunz, R., Fey, M., Jaeger, M., Mayer, A., Hammer, J. W., Staudt, G., Harissopulos, S., \& Paradellis, T. 2002, ApJ, 567, 643

Larson, R. B. 1998, MNRAS, 301, 569

Limongi, M., \& Chieffi, A. 2002, PASA, 19, 246

Limongi, M., \& Chieffi, A. 2003, ApJ, in press (astro-ph/0304185)

Limongi, M., Straniero, O., \& Chieffi, A. 2000, ApJS, 129, 625

Matteucci, F., \& Greggio, L. 1986, A\&A, 154, 279

Prantzos, N., \& Silk, J. 1998, ApJ, 507, 229

Salpeter, E. E. 1955, ApJ, 121, 161

Scalo, J. M. 1986, FCPh, 11, 1

Timmes, F. X., Woosley, S. E., \& Weaver, T. A. 1995, ApJS, 98, 617

Woosley, S. E., \& Weaver, T. A. 1995, ApJS, 101, 181

Yong, D., \& Lambert, D. L. 2003, PASP, 115, 22 